\title{
Convergence Towards Next Generation Wireless Networks
}

\author{
Ritesh C Sadiwala \\ Senior Engineer, Indian Telephone Industries \& Research Scholar, RKDF University Bhopal
}

\begin{abstract}
The communications sector is undergoing significant changes, with the emergence of a number of platforms available to provide a different range of services. Some of these platforms are complementary, others are competitive, or can provide a valid substitute for some of the services provided. Till now, the most important communications platform in most of the countries is the public switched telecommunication network (PSTN), which provides access to all households and buildings across most countries. This paper focuses on the area where the most significant changes are taking place in the communications sector. This paper gives a description of the wire line and wireless (NGN) networks, its basics, its transformation to wireless technology, need for convergence towards next generation networks and the future of NGN networks. The focus is on the next generation access and core networks, it is because they are changing the public switched telecommunication networks, the changes taking place in the PSTN are considerable. For over 100 years copper has been used as the transmission technology in the local loop to connect each home and building to the telecommunication network. Copper is increasingly being replaced by fibre in the local loop while packet-based technology using the Internet Protocol is replacing existing circuit-based switching technologies. This paper describes the need of wireless technology, why and how it becomes essential in terms of technological, social and economic need of the society. It tells us about how the circuit switched technology has been switched to IP technology and how the PSTN networks, which are circuit switched will be converted to packet-based networks These changes affect existing regulations and policy frameworks, convergence, investment and choice in the market place. Finally it concludes with the latest developments, which are going on in Wireless systems.

Index Terms - PSTN, NGN, Convergence, LTE
\end{abstract}

\section{INTRODUCTION}

Communication networks have become a key economic and social infrastructure in today's economic scenario. The network infrastructure supports all economic sectors, which is crucial to the national and international exchange of goods and services, and acts as a main catalyst in changing economic interrelationships through rapid technological change and the proliferation of a range of new services. With the development of the Internet the role of the communication networks has evolved and their importance has increased. The advent of higher access speeds, in many cases symmetric speeds, available to business and to residential subscribers, has also increased the role of communication infrastructures by expanding the available range of services. High speed networks are increasingly helping resolve ongoing societal concerns in areas such as the environment, health care and education, and are increasingly playing a role in social networking. However, for the potential of new network technologies to be realised, the market will require that these networks have universal, or close to universal coverage. The full potential of networks is only likely to be achieved where markets are effectively competitive and solutions have been implemented which ensure adequate coverage to most of the geographic areas. in the telecommunication sector has increased in recent years to a large extent by the high demand for broadband data access, broadband being one of the latest technologies with the fastest growth in penetration rates. Technological innovation, stimulated through digitalisation, has been a major factor in driving change in the communications market. This innovation is reducing costs and enhancing the capability of networks to support new services and applications. A key innovation which is expected to bring further significant changes in the communications market is the transformation from circuit-based public switched telecommunication networks to packet-based networks using the Internet Protocol, so-called next generation networks (NGN). NGN is expected to completely reshape the present structure of communication systems and access to the Internet. The present structure of vertically independent, although interconnected, networks may be transformed into a horizontal structure of networks based on Internet Protocol. The developments in new communication structures and the impetus they are expected to give to the present process of convergence in networks, services and terminals are expected to grow to a larger extent in future. In particular, convergence and the development of the NGN may require a review of a number of elements of the present structure of economic regulation of communication markets, in order to ensure that regulation allows the potential benefits of these technologies to rapidly diffuse in economies and societies. Convergence, by changing service boundaries, service characteristics and stimulating the offer of new services, may require that new markets are regulated differently than existing ones, and this will be possible only by enhancement and development of Next Generation Wireless networks. 


\section{ABOUT PSTN}

The public switched telephone network (PSTN) is the network of the world's public circuit-switched telephone networks. It consists of telephone lines, fiber optic cables, microwave transmission links, cellular networks, communications satellites, and undersea telephone cables, all inter-connected by switching centers ,thus allowing any telephone in the world to communicate with any other. Originally a network of fixed-line analog telephone systems, the PSTN is now almost entirely digital in its core and includes mobile as well as fixed telephones. Historically, incumbent operators typically ran one network - the Public Switched Telephone Network (PSTN). The PSTN was designed to carry voice when voice was the only communication carried. As demand for data communications developed the incumbents adapted their networks to also carry data traffic. However, typically, rather than replacing the PSTN operators typically built new networks that they ran in parallel - which is called the overlay network. These new overlay networks were designed specifically to carry data traffic. As network technology continued to develop, the number of networks multiplied in step. As a result, today, many operators run typically 5-10 different network platforms (ATM, IP, Frame Relay, ISDN, PSTN, $\mathrm{X} .25$ etc.). The problem with this multi-network approach is that it has created a web of complexity resulting in management complexity, operational inefficiencies, smaller economize of scale, maintenance issues, and duplicating capex.

One such PSTN switch as shown in Figure 2.1 is of Alcatel Lucent OCB-283, which was used by BSNL and MTNL, TATA, etc operators presently to run their landline and broadband network. The 1000 E10 (OCB283) exchange, also known by its shorter name as the E10 (OCB283), is designed for evolving networks and the need to rationalize equipment operation. Its modular architecture means that new services can be added and processing capacity can be increased without interrupting operation of the exchange. OCB 283 is digital switching system which supports a variety of communication needs like basic telephony, ISDN, interface to mobile communication, data communication etc. Telecommunications networks are constantly changing. The rapid growth of the digital network, mobile network and intelligent network and the proliferation of new services constantly being offered to subscribers mean that equipment must be continuously adapted to new requirements. The 1000 E10 (OCB283) exchange, also known by its shorter name as the E10 (OCB283), is designed for evolving networks and the need to rationalize equipment operation. Its modular architecture means that new services can be added and processing capacity can be increased without interrupting operation of the exchange. The E10 (OCB283) is a multipurpose exchange. It can be used for a specific function as well as to combine several applications in the same equipment. The multipurpose properties of the E10 (OCB283) mean that it can be used in widely varying contexts. The E10 (OCB283) configuration provide solutions for the following:

a) The application for which it is intended (local switching center, international switching center, etc.)

b) The environment (urban area, rural area).

c) The volume and type of the traffic to be handled.

d) The resources of the telecommunications network to which it is connected. The E10 (OCB283) can be used for all switching applications local switching center, regional or national transit center, international transit center, intelligent network service access point, mobile service access point.

e) The E10 (OCB283) can also provide the STP (signaling transfer point) function of the $\mathrm{N}^{\mathrm{o}} 7$ signaling network.

Figure 2.1. PSTN (OCB-283) Switch in a Wireline network .

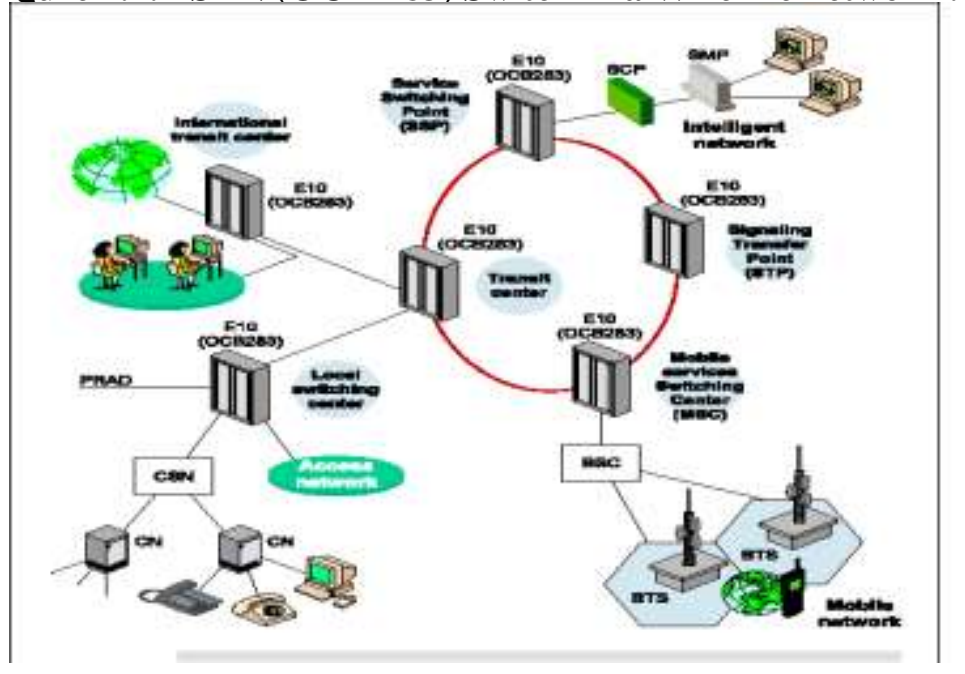


BSC: Base Station Controller

BTS: Base Transceiver Station

CN: Digital Concentrator

CSN: Subscriber Digital Access Unit

PRAD: Primary Rate Access, Directly connected to the exchange

SCP: Service Control Point

SMP: Service Management Point

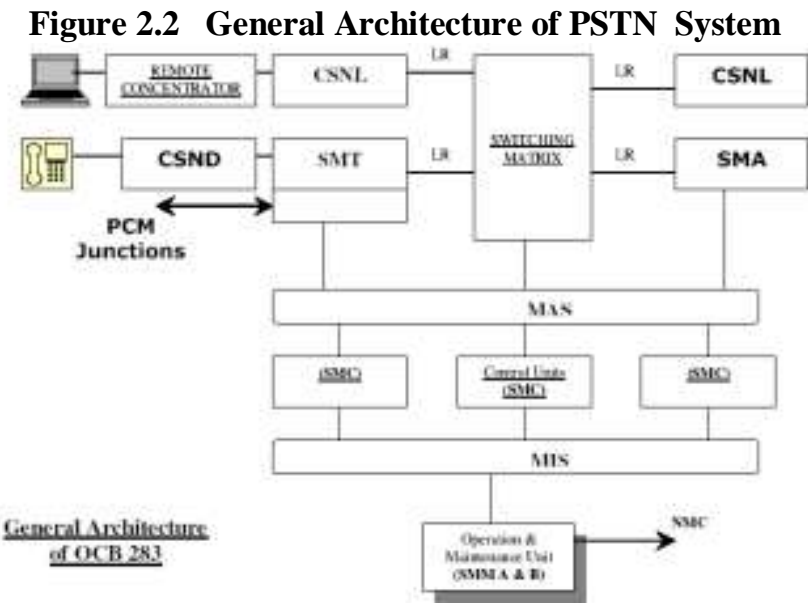

General Architecture of PSTN Switch as shown in Figure 2.2 has three basic subsystems: -

1)Subscriber Access Subsystem

2) Connection and Control Subsystem

3) Operation and Maintenance Subsystem

\section{1) SUBSCRIBER ACCESS SUBSYSTEM}

This is treated as an independent entity. Every Subscriber Connecting Equipment Rack is given a Signaling Point Number to operate in Common Channel Signaling mode with rest of the exchange Subsystems.

\section{2) CONNECTION AND CONTROL SUBSYSTEM}

This unit includes control functions and connection and switching Equipments. Control Functions has common control equipments which process, monitor and control the call setup and release. Connection and switching block comprises of Switching matrix equipment for performing digital time switching of speech path. Connection equipments for connecting PCM (DIGITAL) Junctions from other exchanges and RSU's. Auxiliary Equipments for Tones, Frequencies and other auxiliaries for signaling protocol handling.

\section{3) OPERATION AND MAINTENANCE SUBSYSTEM}

This section is used for Operation and Maintenance of exchange by operators. The functional architecture of the PSTN System as shown in Figure 2.3 comprises of following distinct components: 
Figure 2.3 Functional Architecture of PSTN System

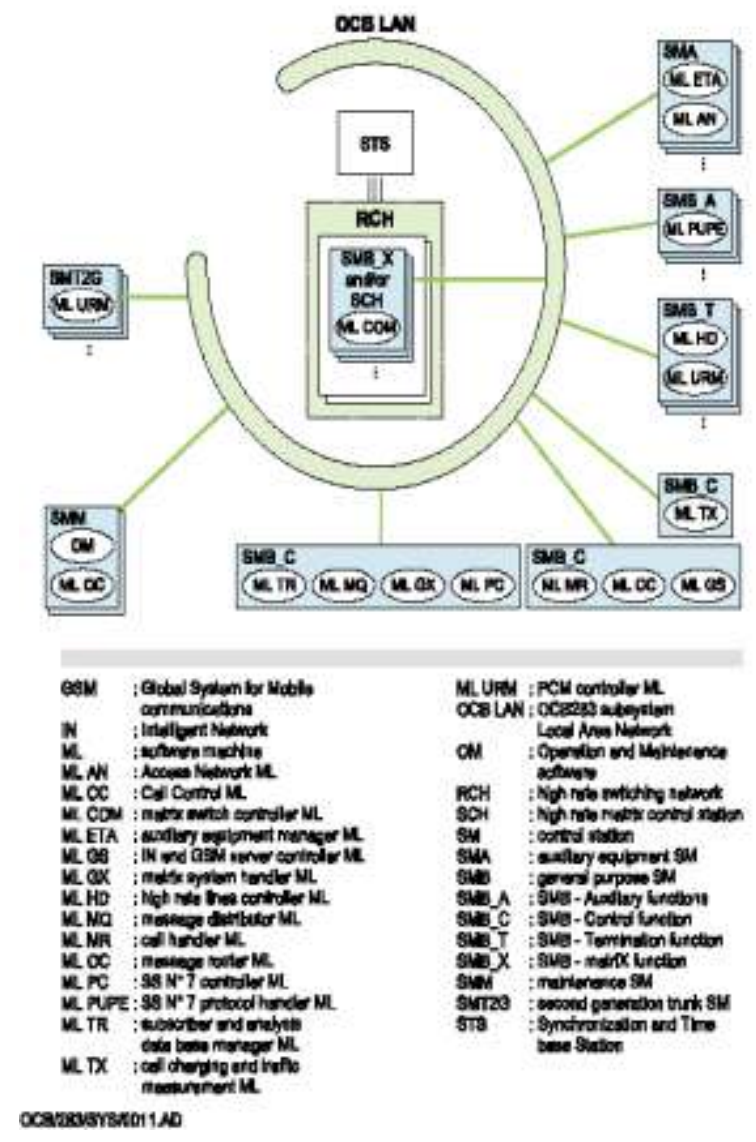

\section{A) SWITCHING NETWORK}

These provides facility for CONNECTING the LRs (internal PCM's) coming from connection units and performs Switching Operation for Calling Subscriber TS onto called Subscriber TS and vice versa for a two way connection per call of telephony. In an Electronic Stored Programme Control Digital Exchange like OCB 283 , all operation and maintenance activities are performed by a unit called O\&M unit or OMC(Operation and Maintenance Centre).This provides access for Man machine dialogues for the human operators to interact and command the working of Exchange Equipments. The function of the operation \& maintenance subsystems are carried out by the operation \& maintenance software $(\mathrm{OM})$. The operating authority accesses all hardware \& software equipment of the PSTN system via computer terminals belonging to the operation and maintenance subsystems: consoles, magnetic media, and intelligent terminal.

\section{B) CONNECTION UNITS}

These provide facility to connect a subscribers loop or circuits from an external PCM and transfer these speech samples on to selected Time slots called voice channels on a LR link (internal PCM) towards switching matrix and vice versa.

\section{C) CONTROL UNITS}

These units provides control of calls on the basis of Stored programmes. They process the calls on reception of dialled digits from calling subscriber/circuit and take part in handling of call setup and release by processing, monitoring, measuring charging of calls and all other common control functions needed for working of an Automatic Common control Exchange. 


\section{II.I System Architecture of PSTN System}

A PSTN system comprises of following hardware units:

1) Subscriber Access Units (CSNL, CSND, CSED)

2) Trunks and Junction connection Units (SMT)

3) Switching Matrix (SMX)

4) Auxiliary Equipments (SMA)

5) Control Units (SMC)

6) Communication multiplexes (MIS \& MAS Token rings)

7) Time Base generator (STS)

8) Operation and Maintenance Unit (SMM)

The subscriber connection units CSN, SMTs and SMAs are connected to switching network through PCM links. The interchange of messages between SMT, SMX, SMA and control units SMCs takes place on 'MAS' token rings. The control units interchange messages with one another and with SMM on 'MIS' token rings.

The SMM is the O\&M function unit \& is duplicated as SMMA \& SMMB. These are in Pilot/Standby mode.

The SMCs are the units, which hold control functions MR, TR, TX, MQ, PC, GX and these FUNCTIONAL units are in software form and are duplicated except MR, which can be more than two. The duplicated functions work in load sharing mode (except PC which works in Pilot/Standbye mode) hence SMCs can be minimum 2 and maximum 32 as per design.The SMA stations hold the ETA \&PUPE functions and these are also minimum 2 to maximum 32.SMT station which is the interface for the external PCMs is made of duplicated hardware and can handle either 32 PCMs if SMT1G or 128 PCMs if 2G. The SMT's hardware is fully duplicated and functions $\mathrm{P} / \mathrm{R}$ mode

\section{ABOUT NGN}

Next Generation Network (NGN) is a packet-based network able to provide services including Telecommunication Services and able to make use of multiple broadband, QoS-enabled transport technologies and in which service-related functions are independent from underlying transport-related technologies. It offers unrestricted access by users to different service providers. It supports generalized mobility, which will allow consistent and ubiquitous provision of services to users. Next Generation Mobile and Wireless Networks (NGMWNs) plays central role to bring together the users, operators, and application developers. From an enduser's point of view, NGN should consistently present him/her with the same personalized services whatever the network that serves him/her and whatever the terminal technology he/she uses. From applications developers' point of view, NGN offers resources and interfaces Application Programming Interfaces "APIs" through which the applications are deployed. From an operators' point of view, NGN is concerned with provisioning and guaranteeing end to end (e2e) Quality of Service (QoS) in the context of all-IP heterogeneous network. In order to meet with such high expectation, NGN network have gone through many changes to come up optimized network design without incurring major changes to network infrastructure. Next Generation Networks aim to reverse the clock and go back to the simplicity of one single network. NGN is all about deploying one network platform capable of supporting all traffic types while facilitating service innovation simplifying the network and streamlining the support structure NGNs can also be defined in terms of their technical characteristics which are as follows:

(i) IP-based network: A single IP-based core network handling the full range of telecom services. Next generation core networks" generally cover the migration from multiple legacy core networks to IP-based networks for the provision of all services. This means that all information is transmitted via packets. Packets can take different routes to the same destination, and therefore do not require the establishment of an end-to-end dedicated path as is the case for PSTN-based communications.

(ii) Packet-based, multi-purpose: In NGN different kinds of applications can be transformed into packets, labelled accordingly and delivered simultaneously over a number of different transport technologies, allowing a shift from single-purpose networks (one network, one service), to multi-purpose networks (one network, many services). Interworking between the NGN and existing networks such as PSTN, ISDN, cable, and mobile networks can be provided by means of media gateways.

(iii) Separation of transport and service layer: This constitutes the key common factor between NGN and convergence, bringing about the radical change in relationship between network "layers" (transport infrastructure, transport services and control, content services and applications). In next generation networks service-related functions are independent from underlying transport-related technologies as shown in Figure 3.1. The uncoupling of applications and networks allow applications to be defined directly at the service level and 
provide seamlessly over different platforms, allowing for market entry by multiple service providers on a nondiscriminatory basis. Distributed rather than centralized switching, routing and network intelligence enabling remote access, control and maintenance.

Figure 3.1 Separation of Layers in NGN

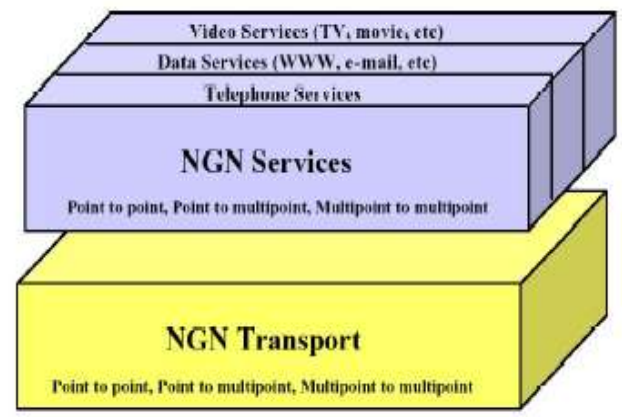

NGNs also have important service characteristics:

a) Continuity - Consumers will be able to continue to use those PSTN services they are used to, with essentially no change.

b) Ease of migration - Consumers will be able to migrate seamlessly to new services offered by the same operator.

c) Single access to multiple services - Driven by the separation of the service layer from the network layer.

d) Innovative new services - New services will have richer functionality (e.g. personalised, location-aware), and reduced time-to-market, since they exploit the distributed intelligence inherent in an NGN.

e) Empowerment - Consumers will have an increased capability to configure and manage services to meet their personal requirements.

\section{III.I Network Architecture of NGN}

In contrast to the circuit-switched model of previous systems, NGN also referred as "Long Term Evolution (LTE)" has been designed to support only packet-switched services. It aims to provide seamless Internet Protocol (IP) connectivity between user equipment (UE) and the packet data network (PDN), without any disruption to the end users' applications during mobility. While the term "LTE" encompasses the evolution of the Universal Mobile Telecommunications System (UMTS) radio access through the Evolved UTRAN (EUTRAN), it is accompanied by an evolution of the non-radio aspects under the term "System Architecture Evolution" (SAE), which includes the Evolved Packet Core (EPC) network. Together LTE and SAE comprise the Evolved Packet System (EPS).EPS uses the concept of EPS bearers to route IP traffic from a gateway in the PDN to the UE. A bearer is an IP packet flow with a defined quality of service (QoS) between the gateway and the UE. The E-UTRAN and EPC together set up and release bearers as required by applications.

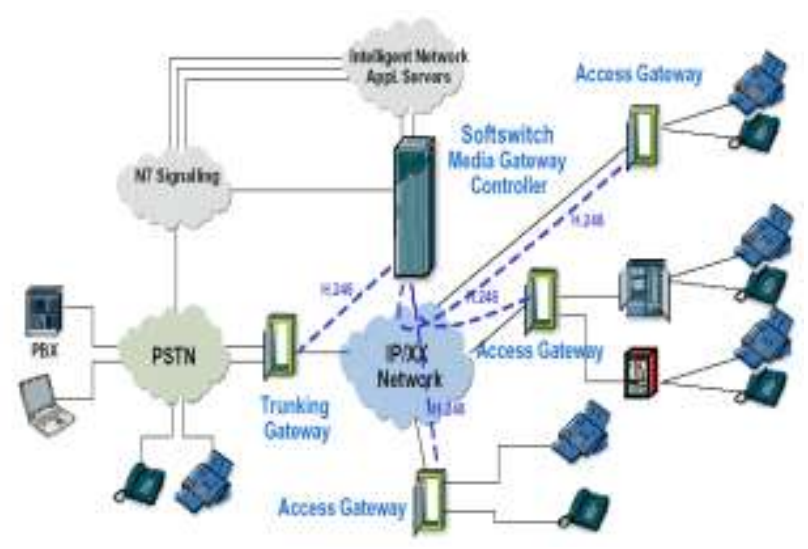

Figure3.2 NGN Network Architecture 
NGN Network Architecture as shown in Figure 3.2 consists of Network Elements, which are as under

1.Packet based networks:-Trend is to use IP based networks over various transport possibilities (ATM, SDH, WDM...).IP based networks must offer guarantees of Quality of Service (QoS) regarding the real time characteristics of voice, video and multimedia.

2. Access Gateways: - It allows the connection of subscriber lines to the packet network converts the traffic flows of analogue access (Pots) or $2 \mathrm{Mb} / \mathrm{s}$ access devices into packets,provides subscriber access to NGN network and services.

3. Trunking Gateways: - It allows interworking between classical TDM telephony network and Packet-based NGN networks, converts TDM circuits/ trunks (64kbps) flows into data packets, and vice versa.

4.Softswitch/MGC: - MGC referred to as the Call Agent or Media Gateway Controller (MGC) which provides the "service delivery control" within the network, in charge of Call Control and handling of Media Gateways control (Access and/or Trunking) via H.248 protocol. It performs signalling gateway functionality or uses a signalling gateway for interworking with PSTN N7 signalling network and provides connection to Intelligent Network /applications servers to offer the same services as those available to TDM subscribers

5. Application Server (AS):- A unit that supports service execution, e.g. to control Call Servers and NGN special resources (e.g. media server, message server).

6. H.248 Protocol :-It is also known as 'MEGACO'is a standard protocol, defined by ITU-T, for signalling and session management needed during a communication between a media gateway, and the media gateway controller managing it.H.248/MEGACO allows to set up, keep, and terminate calls between multiple endpoints as between telephone subscribers using the TDM.

7. SIP :- Session Initiation Protocol in order to handle call establishment, maintenance and termination from packet mode terminals.

8. Signalling Gateway (SG):- A unit that provides signalling conversion between the NGN and the other networks (e.g. STP in SS7).

9.ENUM:-Electronic Numbering is a Protocol that allows to establish a correspondence between the traditional telephone numbering (E.164) and the network addresses related to the packet mode networks ( RFC 2916 "E.164 number and DNS" IETF).

10. MPLS:- Multiprotocol Label Switch or protocol that assigns labels to information packets in order to allow the node routers to treat and route flows in the network paths according to established priority for each category.

11.CAC:- Call Acceptance Control function in order to accept/reject traffic in the network that allows guarantee of QoS for services with a Service Level Agreement

12. BGP :- Border Gateway Protocol to negotiate flow routing procedures and capacities across different NGN network domains. 


\section{Functional Architecture Of NGN}

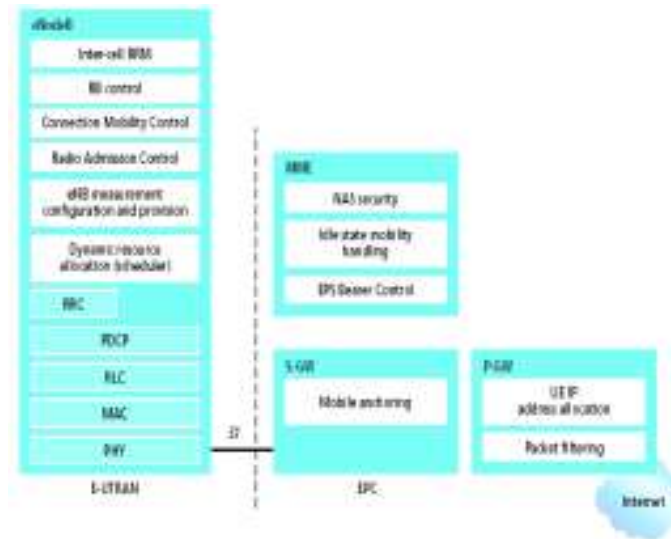

Figure 4.1 NGN Functional Architecture

Functional Architecture of NGN as shown in Figure 4.1 consists of three gateways as under

A) PDN Gateway (P-GW)

B) Serving Gateway (S-GW)

C) Mobility Management Entity (MME)

(A) PDN Gateway (P-GW): - The core network (called EPC in SAE) is responsible for the overall control of the UE and establishment of the bearers.in addition to these nodes, EPC also includes other logical nodes and functions such as the Home Subscriber Server (HSS) and the Policy Control and Charging Rules Function (PCRF). Since the EPS only provides a bearer path of a certain QoS, control of multimedia applications such as VoIP is provided by the IP Multimedia Subsystem (IMS), which is considered to be outside the EPS itself. The PDN Gateway is responsible for IP address allocation for the UE, as well as QoS enforcement and flow-based charging according to rules from the PCRF. It is responsible for the filtering of downlink user IP packets into the different QoS-based bearers. This is performed based on Traffic Flow Templates (TFTs). The P-GW performs QoS enforcement for guaranteed bit rate (GBR) bearers. It also serves as the mobility anchor for interworking with non-3GPP technologies such as CDMA2000 and WiMAX networks.

(B) Serving Gateway (S-GW): - All user IP packets are transferred through the Serving Gateway, which serves as the local mobility anchor for the data bearers when the UE moves between eNodeBs. It also retains the information about the bearers when the UE is in the idle state (known as "EPS Connection Management IDLE" [ECM-IDLE]) and temporarily buffers downlink data while the MME initiates paging of the UE to reestablish the bearers. In addition, the S-GW performs some administrative functions in the visited network such as collecting information for charging (for example, the volume of data sent to or received from the user) and lawful interception. It also serves as the mobility anchor for interworking with other 3GPP technologies such as general packet radio service (GPRS) and UMTS.

(C) Mobility Management Entity (MME): - The Mobility Management Entity (MME) is the control node that processes the signaling between the UE and the $\mathrm{CN}$. The protocols running between the $\mathrm{UE}$ and the $\mathrm{CN}$ are known as the Non Access Stratum (NAS) protocols. The main functions supported by the MME can be classified as

(i) Functions related to bearer management - This includes the establishment, maintenance and release of the bearers and is handled by the session management layer in the NAS protocol.

(ii) Functions related to connection management - This includes the establishment of the connection and security between the network and UE and is handled by the connection or mobility management layer in the NAS protocol layer

\section{Convergence and NGN}

Convergence in network technologies services and in terminal equipment is at the basis of change in innovative offers and new business models in the communications sector. The utilization of the term "convergence" represents the shift from the traditional "vertical silos" architecture, i.e. a situation in which different services were provided through separate networks (mobile, fixed, CATV, IP), to a situation in which communication services will be accessed and used seamlessly across different networks and provided over multiple platforms, in an interactive way. Today's consumers demand is for communications: one-way (video, traffic reports, location, etc,), two-way (traditional voice, e-mail, etc.), symmetrical (traditional voice calls, emails, etc) or asymmetrical (file downloads, movies, etc.). The communications may be fixed, mobile, 
interactive, or unidirectional. With the technology and the move to IP protocol, all of these features can be handled in a few devices and networks - maybe only one. The full integration and convergence of the networks is only beginning to emerge.

\section{V.I WHAT IS CONVERGENCE}

The path towards convergence was led mainly by the increasing digitalisation of content, the shift towards IP-based networks, the diffusion of high-speed broadband access, and the availability of multi-media communication and computing devices. Convergence is taking place at different levels:

(i) Network convergence - It is driven by the shift towards IP-based broadband networks. It includes fixedmobile convergence and 'three-screen convergence' (mobile, TV and computer).

(ii) Service convergence - It enables stemming from network convergence and innovative handsets, which allows the access to web-based applications, and the provision of traditional and new value-added services from a multiplicity of devices.

(iii)Industry/market convergence - It brings together in the same field industries such as information technology, telecommunication, and media, formerly operating in separate markets.

(iv) Legislative, institutional and regulatory convergence - It includes protocols taking place between broadcasting and telecommunication regulation. Policy makers are considering converged regulation to address content or services independently from the networks over which they are provided (technology neutral regulation).

(v) Device convergence -It helps devices to include together a microprocessor, a screen, storage, input device and some kind of network connection so that together they provide multiple communication functions and applications.

(vi)Converged user experience:-It makes unique interface between end-users and telecommunications, new media, and computer technologies. The process towards convergence has been based on an evolution of technologies and business models, rather than

a revolution. This process has led to entry of new players into the market, increasing competition among players operating in different markets and the necessity for traditional operators to co-operate with companies previously in other fields. As a result, convergence has become an important part for the telecommunication sector.

Table 5. Comparison between existing network and IP-based converged network

\begin{tabular}{|c|c|}
\hline $\begin{array}{c}\text { Existing } \\
\text { Networks }\end{array}$ & $\begin{array}{c}\text { Next generation converged } \\
\text { Networks }\end{array}$ \\
\hline $\begin{array}{l}\text { Single purpose } \\
\text { networks }\end{array}$ & Multi-purpose networks \\
\hline $\begin{array}{l}\text { PSTN, cellular, } \\
\text { broadcast }\end{array}$ & $\begin{array}{l}\text { IP network (providing voice, } \\
\text { video and mobile services) }\end{array}$ \\
\hline Narrowband & Broadband \\
\hline Vertical Silos & $\begin{array}{l}\text { Destroys } \\
\text { compartmentalization } \\
\text { i.e,Traditional boundaries } \\
\text { between industry segments } \\
\text { (e.g., telephony, cableTV, } \\
\text { broadcasting, wireless) are } \\
\text { blurring and Need to re-think } \\
\text { market definitions (product } \\
\text { definition and geographic } \\
\text { boundaries definition) }\end{array}$ \\
\hline $\begin{array}{l}\text { Network-service } \\
\text { link }\end{array}$ & $\begin{array}{l}\text { New services and content } \\
\text { developed independently of the } \\
\text { network }\end{array}$ \\
\hline $\begin{array}{l}\text { Operators } \\
\text { control services } \\
\text { to end users }\end{array}$ & Increased consumer control \\
\hline
\end{tabular}




\section{NGN Drivers and Its Impact on Telecom Sector}

NGN is an evolutionary process and it can be expected that operators will take different migratory paths, switching to NGN while gradually phasing out existing circuit networks, or building a fully-IP enabled network from the outset. Telecommunication operators across the world have been faced with a decline in the number of fixed-line telephone subscribers, coupled with a decrease in average revenue per user (ARPU), as a result of competition from mobile and broadband services. Traditional sources of revenue (voice communications) have declined rapidly and fixed-lines operators are subject to an increase in competitive pressure in the market to lower tariffs and offer innovative services. This has generated pressure from the investors' community to decrease the cost and complexity of managing multiple legacy networks, by disinvesting from non-core assets and reducing operational and capital expenses. The investment in developing NGN is motivated by several factors, which are given below

Table 6. Reasons for NGN Networks

\begin{tabular}{|c|c|c|}
\hline $\begin{array}{l}\text { TECHNOLOGICAL } \\
\text { REASONS }\end{array}$ & $\begin{array}{l}\text { ECONOMIC } \\
\text { REASONS }\end{array}$ & SOCIAL REASONS \\
\hline $\begin{array}{l}\text { Obsolescence of legacy } \\
\text { networks, plus cost and } \\
\text { complexity in managing } \\
\text { multiple } \\
\text { networks. }\end{array}$ & $\begin{array}{l}\text { Erosion of } \\
\text { fixed line voice } \\
\text { call } \\
\text { revenues. }\end{array}$ & $\begin{array}{l}\text { Demand for innovative, } \\
\text { high bandwidth, services } \\
\text { (HDTV, VoIP, etc). }\end{array}$ \\
\hline $\begin{array}{l}\text { Lower capital and } \\
\text { operational expenses. }\end{array}$ & $\begin{array}{l}\text { Competitive } \\
\text { pressure from } \\
\text { new entrants in } \\
\text { high-margin } \\
\text { sectors of the } \\
\text { market }\end{array}$ & $\begin{array}{l}\text { Demand for more } \\
\text { targeted or } \\
\text { personalised content (on- } \\
\text { demand multimedia } \\
\text { services, mobility). }\end{array}$ \\
\hline $\begin{array}{l}\text { Increased centralization } \\
\text { of routing, switching and } \\
\text { transmission, lower } \\
\text { transmission costs over } \\
\text { optical networks. }\end{array}$ & $\begin{array}{l}\text { Saturation of } \\
\text { both Fixed and } \\
\text { Mobile } \\
\text { telephone } \\
\text { services. }\end{array}$ & $\begin{array}{l}\text { Demand for increased } \\
\text { interactivity: possibility } \\
\text { to interact actively with } \\
\text { the service, growing } \\
\text { interest for user-created } \\
\text { content. }\end{array}$ \\
\hline $\begin{array}{l}\text { IP-based networks } \\
\text { enable the } \\
\text { provision of cheaper } \\
\text { VoIP } \\
\text { services as a } \\
\text { replacement for } \\
\text { PSTN voice services. }\end{array}$ & $\begin{array}{l}\text { Retain and } \\
\text { expand users' } \\
\text { base } \\
\text { lower customer } \\
\text { churn }\end{array}$ & $\begin{array}{l}\text { Demand for evolved and } \\
\text { more } \\
\text { flexible forms } \\
\text { communications, } \\
\text { including instant } \\
\text { messaging, video- } \\
\text { conferencing, P2P, etc. }\end{array}$ \\
\hline $\begin{array}{l}\text { IP-based networks } \\
\text { enable the } \\
\text { provision of a wider } \\
\text { range of } \\
\text { services, and allow } \\
\text { bundling of services } \\
\text { (triple and quadruple } \\
\text { play). }\end{array}$ & $\begin{array}{lr}\text { Ability to } \\
\text { expand into } \\
\text { new } \\
\text { market } \\
\text { segments }\end{array}$ & $\begin{array}{l}\text { Business demand for } \\
\text { integrated services, } \\
\text { particularly in case of } \\
\text { multi-national structures. }\end{array}$ \\
\hline $\begin{array}{l}\text { Evolution } \\
\text { convergence of } \\
\text { terminal equipment. }\end{array}$ & $\begin{array}{l}\text { Possibility of } \\
\text { investment in } \\
\text { phased } \\
\text { approach for } \\
\text { investment, } \\
\text { initially } \\
\text { targeting more } \\
\text { densely } \\
\text { populated } \\
\text { areas, and then } \\
\text { gradually } \\
\text { expanding in } \\
\text { other areas }\end{array}$ & $\begin{array}{l}\text { Guaranteeing a flexible } \\
\text { and secure access to } \\
\text { centralized resources and } \\
\text { intelligence. }\end{array}$ \\
\hline
\end{tabular}




\section{MIGRATION TOWARDS NGN}

Next Generation Networks do not represent a specific technology but rather a system and a market concept enabled by combination of different advance technologies. The basic features of NGN are range of access platforms, independence and range of service architecture, different migration paths and timing.

The drivers for migrating to NGN are different for different players viz. reducing their network infrastructure and maintenance costs, enabling faster service deployment for the provisioning of enhanced services and therefore creating new sources of revenue. The high flexibility, low cost and wide support throughout the world for the Internet Protocol makes it the best option for building NGNs, even though it has some limitations that need to be overcome for example the lack of guaranteed QoS and security requirements. Network operators will potentially choose a different migration path depending on their existing assets. Each path may therefore involve different technologies and happen at different pace. For circuit-switched network operators, a multi-service network for converged services and more revenues represents the main driver for the migration to NGNs. However, a migration also imposes various direct and indirect costs including network upgrades, staff retraining costs, organization process changes, etc. Moreover, some circuit-switched networks specially the cellular mobile systems are still in a very good state and provide quality telephony services, which are currently difficult to replicate on a large-scale in IP based networks.

Packet-switched data networks rely on several technologies and a number of protocol stacks are used depending on the service delivered and the functionality offered by each protocol, e.g. "IP over SDH over DWDM", or "IP over ATM over SDH over DWDM", or "IP over Ethernet over SDH over DWDM". The migration towards NGN for these types of networks means a simplification of the network and more flexibility. Building an NGN network also means that the network needs to support converged services including voice and real-time applications. The migration of packet-based networks towards NGN can follow several strategies that could possibly be combined. A migration to NGN could also involve a move towards the next version of the Internet Protocol, IPv6.

NGN concept also implies many different types of "Convergence";

1. PSTN/ISDN convergence with IP based networks.

2. Fixed - Mobile convergence (FMC).

3. Broadcast (Cable) - Telecommunications convergence.

4. Web based services convergence

The convergence towards Next Generation Networks also requires that customers of different market players, using different network technologies, can communicate with each other and access resources on another market player's network. This requires the interworking of different Naming, Addressing and Numbering systems. Two main standardised numbering solutions, which can be discussed for NGN, are ENUM and TIPHON.

In order to convert a PSTN network to NGN network three parts has to convert from PSTN to NGN. Firstly call control mechanism of PSTN has to be converted to soft switch where all the functionalities are governed by a single call server, secondly circuit based switching has to be replaced by IP based switching governed by MPLS(Multi protocol Label Switching) where switching is done by routers so that the congestion of the data should be minimal and data can be forwarded by any path, several paths are designed by routers and the call/data will move by the shortest path available and with fastest speed. Thirdly PSTN interfaces are changed by NGN gateways. These gateways work on standard protocols as defined by industry standard so as to reduce the time taken by the data to reach the destination as well as they are supported by IP platform. Further the TDM transport network has been converted to a common IP MPLS platform in order to have a common platform for transporting all types of data and information. The designed migration of PSTN to NGN is shown in Figure 7.1 


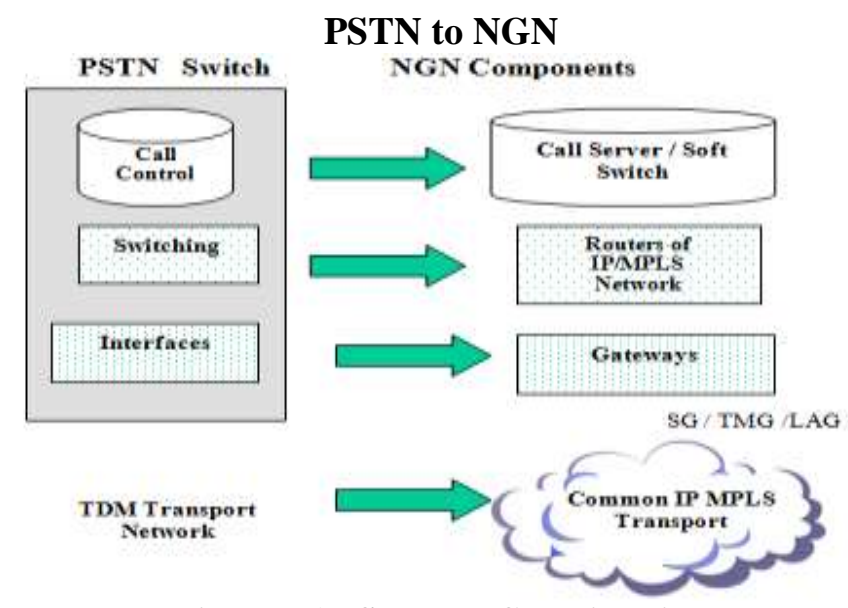

Figure 7.1 PSTN to NGN Migration

A fully converged NGN Architecture is presented in Figure 7.2, which shows a converged network along with its protocols and its key IMS components

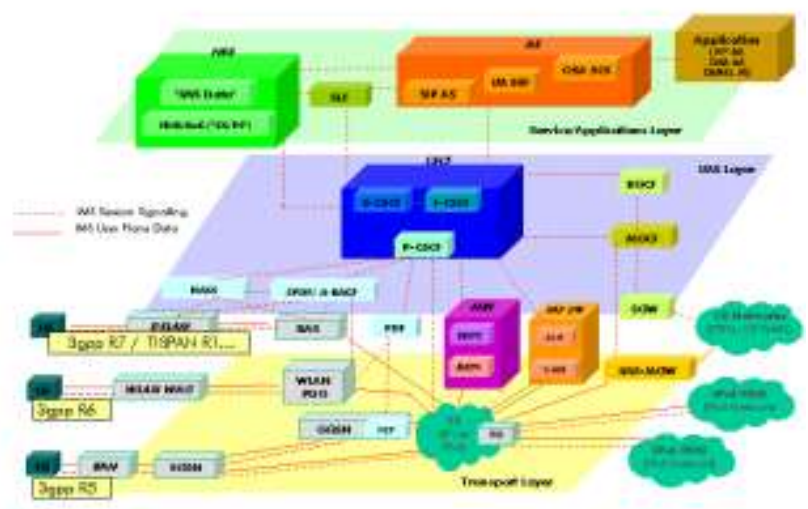

Figure 7.2 A Fully Converged NGN Network

The description of IMS components of an NGN network is given below

Table 7. IMS Key Components

\begin{tabular}{|c|c|c|}
\hline Network Function & Role & Origin \\
\hline $\begin{array}{l}\text { Call Session Control } \\
\text { Function (CSCF) }\end{array}$ & $\begin{array}{l}\text { Handles } \\
\text { registration } \\
\text { of end points, } \\
\text { routing of } \\
\text { SIP signaling } \\
\text { messages }\end{array}$ & $\begin{array}{lr}\text { SIP Proxy } & \text { Servers, } \\
\text { Developed } & \text { from } \\
\text { scratch } & \end{array}$ \\
\hline $\begin{array}{l}\text { Homer Subscriber } \\
\text { Server (HSS) }\end{array}$ & $\begin{array}{l}\text { One-stop } \\
\text { database for } \\
\text { user } \\
\text { information }\end{array}$ & $\begin{array}{l}\text { HLR from mobile } \\
\text { network }\end{array}$ \\
\hline $\begin{array}{ll}\text { Media } & \text { Gateway } \\
\text { Control } & \text { Function } \\
\text { (MGCF) } & \end{array}$ & $\begin{array}{l}\text { Softswitch } \\
\text { Media } \\
\text { Gateway } \\
\text { Control }\end{array}$ & Softswitch \\
\hline $\begin{array}{ll}\text { Application } & \text { Server } \\
\text { (AS) } & \end{array}$ & $\begin{array}{l}\text { Provide } \\
\text { service logic } \\
\text { for } \\
\text { applications }\end{array}$ & $\begin{array}{lr}\text { Feature } & \text { Servers, } \\
\text { Feature sets } & \text { from } \\
\text { Softswitches } & \end{array}$ \\
\hline $\begin{array}{l}\text { Policy Decision } \\
\text { Function (PDF) }\end{array}$ & $\begin{array}{l}\text { Provides } \\
\text { QoS }\end{array}$ & $\begin{array}{l}\text { Existing } \\
\text { Managers, } \\
\text { scratch }\end{array}$ \\
\hline
\end{tabular}




\section{CONCLUSION AND FUTURE WORK}

This paper gives an indepth knowledge of PSTN and NGN systems. An ongoing research is presented for the wireline and wireless systems, describing the features of both the systems. This paper summarizes the key challenges and benefits for Service Providers (SPs) that plan to migrate from PSTN to NGN

* The PSTN convergence is necessary to help SPs lower their overall cost structure. Migrating the PSTN traffic to NGN will increase the utilization in NGN and help SPs' lower NGN cost structure.

* SPs are rolling out major broadband access transformation programs. A strategy that couples the PSTN migration and the access transformation will help minimize the PSTN migration costs.

* A seamless co-existence of NGN with some consolidated/integrated parts of the PSTN is a more realistic target over the short and intermediate terms. A key limitation in the delivery of hybrid services is the distribution of point codes in NGN.

* A staged migration to a target converged NGN architecture is recommended. Further the need of convergence is explained, showing why now it has became necessary to shift from PSTN to NGN by giving the reasons on social, technological and economic front. This paper is a research work of the practical systems of communication. A lot of detailed work has been given on NGN systems in this paper but some issues such as on secure interoperability between and among the networks, the pricing of both the wholesale and retail services, and on the open or closed architecture of the systems are remaining which will be covered as future work.

In conclusion, we believe that maintaining the status quo has increasingly become cost prohibitive for the service providers. As the number of PSTN lines continues to drop as a result of wireless substitution and VolP over broadband adoption, service providers need to transform the legacy PSTN network with new technologies to lower their cost structure. NGN has emerged as the prevalent consensus in the telecom industry. Strategies that couple the PSTN migration with broadband access transformation can significantly reduce the cost of migration and is proven to result in a positive business case. Therefore, we conclude that the industry has reached the tipping point for large-scale migrations of the PSTN services to NGN systems.

\section{REFERENCES}

[1] Dave Wisely, "IP for 4G", Wiley India, 2011.

[2] International Telecommunication Union, Ruling the New and Emerging Markets in the Telecommunications Sector, Challenges: The Emergence of Next Generation Networks Background Paper, NGN/01, 23 March 2006, p. 14.

[3] International Telecommunication Union, Interconnection in an NGN Environment, Background Paper, NGN/02, 23 March 2006.

[4] International Telecommunication Union, What Rules for Universal Service in an IP enabled NGN Environment? Background Paper, NGN/03, 23 March 2006.

[5] Telecom Regulatory Authority of India, Consultation Paper on Issues Pertaining to Next Generation Networks (NGN), 12 January 2006, Consultation Paper No: 2/2006.

[6] International Telecommunication Union, NGN Overview, International Conference for Regulators, Dubai, United Arab Emirates, 7 February 2007.

[7] Organization for Economic Cooperation and Development, “Convergence and Next Generation Networks",International Conference for OECD community, Seoul,Korea, June 2008.

[8] Telecom Regulatory Authority of India, "Issues pertaining to Next Generation Networks", National Conference of Telecom Regulators, Mumbai,India, January 2011.

[9] Dr Jingming Li Salina, "Next Generation Networks", John Wiley \&Sons Ltd.,2008

[10] Tsuguo Kato, "PSTN, Timeline History" by Icon Group International, 2010. 